\title{
Cooking losses of long-chain $n-3$ fatty acid in seafood commonly eaten in the UK
}

\author{
M. V. P. James ${ }^{1}$, K. D. Flint ${ }^{1}$ and C. H. S. Ruxton ${ }^{2}$ \\ ${ }^{1}$ Young's Seafood Ltd, Ross House, Wickham Road, Grimsby DN31 3SW, UK and ${ }^{2}$ Nutrition Communications, \\ Front Lebanon, Cupar KY15 4EA, UK
}

Differences in the types of fatty acids (FA) present in oily and white fish have implications for loss of very-long-chain $n$-3 fats (LC-PUFA) during cooking. While the majority of LC-PUFA in oily fish is in the relatively stable form of TAG, most LC-PUFA in white fish are present as phospholipids, which are vulnerable to oxidation at higher temperatures ${ }^{(1)}$. The present study analysed FA in three species of fish, in the raw and cooked states, to quantify cooking losses of LC-PUFA.

Whole de-boned fillets of Alaskan Pollock (Theragra chalcogramma), cod (Gadus morhua) and Atlantic salmon (Salmo salar) were homogenised and split into six standardised samples. Three samples were analysed in the raw state for FA content using GC and comparison with a FA reference set. The other three samples were sealed in plastic pouches and cooked in a pan of boiling water until an internal temperature of $72^{\circ} \mathrm{C}$ was achieved for $2 \mathrm{~min}$. After cooling, the samples were analysed for FA content as for the raw samples. The mean findings for the raw and cooked fish $(\mathrm{g} / 100 \mathrm{~g})$ are presented in the Table:

\begin{tabular}{lccc}
\hline & Alaskan pollack & Cod & Atlantic salmon \\
\hline Raw & & & \\
Total fat & 0.36 & 0.70 & 14.4 \\
LC-PUFA & 0.17 & 0.28 & 2.34 \\
DHA & 0.09 & 0.17 & 0.79 \\
EPA & 0.08 & 0.11 & 1.15 \\
Cooked & & & \\
Total fat & 0.33 & 0.72 & 14.5 \\
LC-PUFA & 0.12 & 0.26 & 2.34 \\
DHA & 0.06 & 0.15 & 0.78 \\
EPA & 0.06 & 0.10 & 1.15 \\
LC-PUFA retained $(\%)$ & 72 & 92 & 100 \\
\hline
\end{tabular}

Atlantic salmon, a high-fat oily fish, had a LC-PUFA content that was similar whether in the raw or cooked state. This result agrees with other research findings ${ }^{(2)}$. In contrast, LC-PUFA losses in the white fish were $28 \%$ for pollock and $8 \%$ for cod. More research is needed for other species and different cooking methods. LC-PUFA losses as a result of cooking should be acknowledged in government advice to consumers and when making on-pack claims about the average LC-PUFA content of seafood. Although white fish contributes to overall LC-PUFA intakes, the cooking losses can be greater than for high-fat species of oily fish.

1. Mai J, Shimp J, Weihraunch J et al. (1978) J Food Sci 43, 1669-1674.

2. Al-Saghir S, Thurner K, Wagner KH et al. (2004) J Agric Food Chem 52, 5290-5296. 